\title{
DENSE CLOUDS NEAR THE CENTRAL ENGINE OF ACTIVE GALACTIC NUCLEI
}

\author{
R. SIVRON AND S. TSURUTA \\ Physics Department, Montana State University, Bozeman MT 59717 \\ Received 1992 April 16; accepted 1992 July 14
}

\begin{abstract}
A model is presented which assumes the existence of cold dense clouds near the central engine of active galactic nuclei (AGNs). The effects of such clouds on the observed spectrum are explored. It is shown that this model is consistent with the complicated observed spectra and variability behavior of most extensively studied Seyfert nuclei. The results are compared with other proposed models. The existing observational evidence appears to support the "cloud-model."

Subject headings: dense matter - galaxies: active - galaxies: nuclei - galaxies: Seyfert - quasars: general
\end{abstract}

\section{INTRODUCTION}

The generally power-law spectrum of a class of AGNs represented by radio-quiet quasars and Seyfert nuclei is consistent with a synchro-Compton model where the primary synchrotron radiation is modified through Compton scattering by a nonthermal electron-positron pair plasma near a supermassive black hole (e.g., Fabian et al. 1986). Recently additional fine structures, such as the Fe line-edge feature and hard X-ray bump, have been observed in many of Seyfert nuclei (e.g., Nandra et al. 1991 and references therein). These observed characteristics are naturally explained by the "twocomponent" model which consists of the hot nonthermal plasma responsible for the Comptonization of the primary synchrotron radiation and the cool plasma which reprocesses and reflects the nonthermal radiation (e.g., Guilbert \& Rees 1988, hereafter GR88; Lightman \& White 1988, hereafter LW88). The possibility for the cold matter to lie near the central region is supported by the simultaneous rapid variations of the X-ray continuum and $\mathrm{Fe}$ line fluxes, to within $\sim 300 \mathrm{~s}$, recently detected in Seyfert nucleus NGC 6814 (Kunieda et al. 1990). The cold component is envisioned to be either in a form of a thin disk (or slab) or an assembly of clouds distributed over a three-dimensional configuration (LW88, GR88). For convenience, we shall call the former kind "the disk model" and the latter "the cloud model." In order for the cold clouds to exist near the central engine they must be sufficiently dense, and some physical pressure is required to confine them. This pressure may be supplied by the equipartition magnetic fields (Rees 1987; GR88; Celotti, Fabian, \& Rees 1992, hereafter CFR92).

The spectral and temporal behavior of the outcoming radiation depends on the geometry of the radiating and refecting plasmas. So far, numerical calculations of reflection of nonthermal radiation from cold matter have been carried out only for the disk (or slab) geometry (e.g., LW88; George \& Fabian 1991). However, existing observations already appear to favor the cloud model (see $\S 4$, also Tsuruta 1992). In this paper, therefore, we extend the previous work by carrying out numerical calculations of reflection of nonthermal synchroCompton radiation from cool, dense clouds confined in a geometrically defined region.

Section 2 outlines the basic equations and methods adopted in this work. The results are presented in $\S 3$. Discussion and concluding remarks are given in $\S 4$, the last section.

\section{BASIC EQUATIONS}

In deriving our equations the general approaches of GR88, LW88, and CFR92 are followed. Further details are found in Sivron \& Tsuruta 1992 (hereafter ST92). The free parameters used in our work are the total bolometric luminosity $L$, the mass of the black hole $M$, and the total covering factor due to the cloud system $f$.

In this work we consider accretion-powered AGNs radiating close to the Eddington limit, which are generally thought to apply to Seyfert nuclei and radio-quiet quasars. The total luminosity is therefore $L=\eta \dot{M} C^{2}$, where $\eta$ is the efficiency of the process and $\dot{M}$ is the accretion rate. For a continuously smooth and isotropic inflow of matter the accretion rate is $\dot{M}=4 \pi R^{2} n m_{H}\left(v_{\text {ace }} / v_{\mathrm{ff}}\right) \sqrt{2 G M / R}$. The density of the infalling matter is $\sim m_{\mathrm{H}} n(R)$, where $n$ is the average number density and $m_{\mathrm{H}}$ is the mass of one hydrogen atom. (Assuming cosmic abundance this is a reasonable approximation for the mass of an atom.) The infall velocity of the accreted matter is $v_{\mathrm{acc}}$, which is assumed to be proportional to $v_{\mathrm{ff}}$, the free-fall velocity. Hence the minimal optical depth of isotropically infalling matter is determined by $\tau=\int \kappa_{\mathrm{es}} n m_{\mathrm{H}} d R$, which can be expressed as

$$
\tau=10\left(\frac{\eta}{0.1}\right)^{-1}\left(\frac{L}{L_{\mathrm{Edd}}}\right)\left(\frac{R}{R_{g}}\right)^{-1 / 2}\left(\frac{t_{\mathrm{acc}}}{t_{\mathrm{ff}}}\right),
$$

where $R_{g}=2 G M / c^{2}$ is the gravitational radius of the central black hole, and $t_{\mathrm{acc}}$ and $t_{\mathrm{ff}}$ are the accretion and free-fall time scales, respectively (GR88). The Eddington luminosity is $L_{\mathrm{Edd}}=4 \pi G M m_{\mathrm{H}} c / \sigma_{\mathrm{T}}$, where $G, c$, and $\sigma_{\mathrm{T}}$ are the gravitational constant, the speed of light, and the Thomson cross section. When the source is radiating close to $L_{\mathrm{Edd}}$, therefore, $\tau$ will exceed one near the center, and the original power-law emission will be shrouded and reprocessed by the infalling matter. Consequently the final spectrum is expected to deviate from a simple power law (GR88, LW88), as indeed observed by the $\mathrm{X}$-ray satellites such as Ginga (Nandra et al. 1991, and references therein).

When the inflow of matter is not smooth the above equations should be modified. In the two-component cloud model the frequency dependent optical depth of one cloud is

$$
\tau_{\mathrm{v} 1 \mathrm{cld}}=\int_{R}^{R+2 l_{\mathrm{cld}}} d R\left(\kappa_{\mathrm{v}}+\kappa_{\mathrm{KN}}\right) n_{\mathrm{th}} m_{\mathrm{H}},
$$


where $n_{\mathrm{th}} m_{\mathrm{H}}$ is the density of the cloud, $\kappa_{\mathrm{KN}}$ and $\kappa_{v}$ are, respectively, the free-free and Klein-Nishina opacity, $v$ is the frequency, and $l_{\text {cld }}$ is the radius of a cloud. An accurate optical depth that includes the atomic properties and the Einstein coefficients, such as the one presented by Ferland \& Rees (1988), will be included in our subsequent work (ST92).

Because of fast Compton and faster bremsstrahlung cooling most of the shrouding infalling clouds should be relatively cold, but the temperature of the clouds cannot be lower than

$$
T_{\mathrm{cld}}=\left[(1-A) \int_{v_{\min }}^{v_{\max }} d v \frac{L_{v}}{16 \sigma_{B} \pi R^{2}}\left(1-e^{-\tau_{v 1 \mathrm{cld}}}\right) e^{-f(R)}\right]^{1 / 4}
$$

because of the absorption of the primary radiation and the subsequent thermal reemission. Here $A$ is the albedo (per cloud), $\sigma_{\mathrm{B}}$ is the Stefan-Boltzmann constant, and $f(R)$ is the covering factor at $R$, which might be also frequency-dependent. This temperature is reached if $n_{\mathrm{th}}$, the number density of matter in the cold clouds, is high enough so that the bremsstrahlung cooling time satisfies $t_{\text {bremss }} \ll t_{\text {Compton }} \ll t_{\text {synchrotron }}$, where $t_{\text {Compton }}$ and $t_{\text {synchrotron }}$ are the cooling times due to Compton scattering and synchrotron emission, respectively. This high density can result from a confining pressure supplied by the equipartition magnetic fields, $P_{\text {magnetic }}=B^{2} / 16 \pi \sim n m_{\mathrm{H}} G M / R$ (Rees 1987). Then a two-phase structure with dense cold clouds and hot nonthermal plasma can exist. By equating the gas pressure to the confining magnetic pressure we get (GR88, also see LW88)

$$
\frac{n_{\mathrm{th}}}{n}=m_{\mathrm{H}} c^{2}\left(\frac{R}{R_{g}}\right)^{-1}\left(k T_{\mathrm{cld}}\right)^{-1} .
$$

Another important relation between the above variables can be derived from arguments similar to those that lead to equation (1). The accretion rate of the infalling clouds is the product of $n_{\text {cld }}$, the number of clouds per unit volume, the mass of one cloud, the accretion velocity of the clouds, and the surface area of a spherical shell. When the clouds hold most of the infalling mass, their accretion velocity is $\sim v_{\text {acc }}$ so that

$$
\dot{M} \sim \frac{d M_{\mathrm{cld}}}{d t}=\frac{4 \pi l_{\mathrm{cld}}^{3}}{3} 4 \pi R^{2} n_{\mathrm{cld}} n_{\mathrm{th}} m_{\mathrm{H}} v_{\mathrm{acc}} .
$$

One of the assumptions made for the cloud model is that a significant fraction of $\dot{M}$ does not fall into the equatorial plane to form an accretion disk when the clouds are small and dense enough, even when a small amount of angular momentum is present. Therefore, when the time scale for the cold matter to fall into a disk is longer than the typical infall time scale $R / v_{\mathrm{ff}}$, most of the matter will be in the form of clouds. We assume that the typical collision time $t_{\mathrm{coll}} \leq 1 /\left(n_{\mathrm{cld}} \pi l_{\mathrm{cld}}^{2} v_{\mathrm{cld}}\right)$ is equal to the time scale for falling into a disk, where $v_{\text {cld }}$ is the random velocity of the clouds. From this requirement we obtain the following relation between $n_{\text {cld }}$ and $l_{\text {cld, }}$,

$$
n_{\mathrm{cld}} l_{\mathrm{cld}}^{2}<\frac{1}{\pi}\left(\frac{R_{g}}{R^{3}}\right)^{1 / 2}\left(\frac{v_{\mathrm{cld}}}{c}\right)^{-1}
$$

If the main force acting on the clouds is gravitational, $v_{\text {cld }}$ is roughly the Keplerian velocity. Otherwise the explicit dynamics should be considered.

As the input to our spectral calculations, a point source is adopted which emits a power-law spectrum from the innermost region of the central engine. The model has to satisfy the variability requirement imposed by the absence of IR-optical variations during violent X-ray flares, which is noted for Seyfert nucleus NGC 4051 (Done et al. 1990). Therefore, we assume that the IR-optical portion of this primary radiation is effectively absorbed by the extremely small cloudlets in the vicinity of the black hole which are transparent to the higher energy photons, as suggested by CFR92. We shall call these small clouds "the CFR cloudlets." In our model this modified spectrum is used as the input radiation, to be reflected and reprocessed by an assembly of cold, optically thick clouds. These clouds are distributed over a spherical region from $R_{\min }$, just outside the outer boundary of the innermost region with the CFR cloudlets, to some maximum radius $R_{\max }$, which is to be defined shortly (see § 3 ). Since the lower frequency IRoptical portion of the primary synchro-Compton spectrum has been filtered out by the CFR cloudlets, the radiation pressure on the clouds in our region due to free-free absorption is sig. nificantly reduced, and consequently in this region optically thick clouds larger than the CFR cloudlets can exist (see CFR92; ST92).

The outcoming observed spectrum $I_{\text {obs }}$ is calculated from the radiative transfer equation for an assembly of clouds isotropically distributed around the modified synchro-Compton source (ST92)

$$
I_{\text {obs }}(s)=I_{\text {in }}(0) e^{-\tau_{\mathrm{cld}}}+\int_{0}^{\tau_{\mathrm{cld}}} e^{-\left(\tau_{\mathrm{cld}}-\tau_{\mathrm{ccld}}\right)} S_{\mathrm{cld}}\left(\tau_{\mathrm{cld}}^{\prime}\right) d \tau_{\mathrm{cld}}^{\prime} .
$$

Here $I_{\mathrm{in}}(0)$ is the input spectrum, $S_{\text {cld }}$ is the source function, and $\tau_{\text {cld }}$ is the optical depth of the cloud system,

$$
\tau_{\text {cld }}=\int_{s_{0}}^{s} d s^{\prime} \pi n_{\text {cld }}\left(s^{\prime}\right) l_{\text {cld }}^{2}\left(s^{\prime}\right),
$$

where $s$ is the path length variable and $s_{0}$ is $R_{\min }$. Note that $\tau_{\text {cld }}$ is independent of $\tau_{v 1 \text { cld }}$, the optical depth of one cloud, when the clouds are optically thick.

The outcoming spectrum which is due to reflection from the clouds is

$$
\begin{aligned}
I_{\mathrm{ref}} & =\int_{R_{\min }}^{R_{\max }} d R \\
& \times \int_{\theta=0}^{\pi} d \theta\left[2 \pi R^{2} \sin \theta e^{-f(R)}\left[S_{\mathrm{ref}} n_{\mathrm{cld}} \frac{\pi l_{\mathrm{cld}}^{2}(1+\cos \theta)}{4 \pi R^{2}}\right]\right. \\
& \left.\times\left\{\exp \left[-\int_{\theta=0}^{\pi-\operatorname{srcsin}\left(R \sin \theta / R_{\mathrm{mar}}\right)} d \tilde{\theta} \sin \tilde{\theta} R n_{\mathrm{cld}} \pi l_{\mathrm{cld}}^{2}\right]\right\}\right],
\end{aligned}
$$

where $\theta$ is the angle subtended by the central source and the observer at the cloud. The parameter $S_{\mathrm{rer}}\left[n_{\mathrm{ih}}, I_{\mathrm{int}}(0), f\right]$ (see, for example, LW88) is the source function due to reflection from an individual cloud at $R$. For example, the solution of the radiative transfer equation for low energies is given by $S_{\text {ref }}=$ $I_{\text {in }}(0)(1-\sqrt{\epsilon}) /(1+\sqrt{\epsilon}) / R^{2}$, where $\epsilon=\kappa_{v} /\left(\kappa_{v}+\kappa_{\text {es }}\right)$. The freefree opacity $\kappa_{v}$ depends on the density $n_{\mathrm{th}} m_{\mathrm{H}}$ of a cloud, which is derived from equations (3) and (4). The additional effects of the relative orientation of the cloud with respect to the central point source and the observer are taken into account by the method of phases. For example, the decrease in reflection from a single spherical cloud is proportional to $\pi l_{\mathrm{cld}}^{2}(1+\cos \theta)$, similar to the solution for the luminosity of the moon phases. This decrease is crudely of the same order of magnitude as that of a circular slab tilted at an angle of $\theta=0^{\circ}-60^{\circ}$ (George \& Fabian 1991, Fig. 12). The occultation of radiation after it was 
reflected from the cloud is taken into account in the second exponential in equation (6b).

The thermal component of the outcoming radiation due to absorption and reemission by the clouds is calculated from

$$
\begin{aligned}
& I_{\text {thermal }}=\int_{R_{\min }}^{R_{\max }} d R \int_{\theta=0}^{\pi} d \theta\left[\left[n_{\mathrm{cld}} 2 \pi R^{2} \sin \theta\left(F_{\text {thermal }} \pi l_{\text {cld }}^{2}\right)\right.\right. \\
& \left.\quad \times\left\{\exp \left[-\int_{\tilde{\theta}=0}^{\pi-\arcsin \left(R \sin \theta / R_{\mathrm{max}}\right)} d \tilde{\theta} \sin \tilde{\theta} R n_{\mathrm{cld}} \pi l_{\mathrm{cld}}^{2}\right]\right\}\right],
\end{aligned}
$$

where $F_{\text {thermal }}\left[T_{\text {cld }}(R)\right]$ is the thermal radiation flux from the surface of the clouds.

The observed total spectrum is

$$
I_{\text {obs }}=I_{\text {in }}(0) e^{-\tau_{\text {cld }}}+I_{\text {ref }}+I_{\text {thermal }} .
$$

This is a shorthand version of equation (6a). The integral of $I_{\text {obs }}$ gives the total observed luminosity $L$ which should be equal to the integral over the primary spectrum, because we assume that there is no significant amount of viscosity among the clouds.

The covering factor is defined as

$$
f(R)=\int_{R_{\min }}^{R} n_{\mathrm{cld}} \pi l_{\mathrm{cld}}^{2} d R .
$$

Then, $f \equiv f\left(R_{\max }\right)$ gives the total covering factor of the cloud system. The four unknowns $n_{\text {th }}, T_{\text {cld }}, n_{\text {cld }}$, and $l_{\text {cld }}$, which determine $S_{\text {ref }}$ and $F_{\text {thermal }}$, are obtained by solving equations (2), (3), (4), and (7). Once our free parameters $L=4 \pi \int d \nu I_{\text {in }}(0), M$, and $f$ are specified the total spectrum is uniquely determined. For convenience, in our calculations an effectively equivalent choice of $T_{\text {cld }}(L, M, f), n_{\text {cld }}\left(T_{\text {cld }}, M\right)$, and $f$ are adapted as our free parameters (see ST92 for the details).

The integrations in equations (6b) and (6c) are solved numerically. The results, combined with the unabsorbed portion of the input spectrum, can be compared with the slab case where $I_{\mathrm{obs}}=0.5 I_{\mathrm{in}}+S_{\mathrm{ref}}+\pi\left(R_{\max }^{2}-R_{\min }^{2}\right) F_{\text {thermal }}$.

\section{RESULTS}

The basic equations (2), (3), (4), and (7) are solved for the spherically symmetric case. The results show the radial distribution of our characteristic parameters to be $T_{\text {cld }}(R) \propto$ $R^{-1 / 2}, n_{\mathrm{th}}(R) \propto R^{-2}$, and $f(R) \propto \log (R)$ to $R^{1}$. A small covering factor $f=f\left(R_{\max }\right)<1$ and large optical depth per cloud $\tau_{v 1 \text { cld }}>1$ are assumed. The physical implication is that the luminosity is less than the Eddington luminosity. Note that for $f\left(R_{\max }\right)>1$ the central source is heavily attenuated and the radial dependence becomes more complex. The final spectrum was found to be almost insensitive to the radial dependence of $n_{\text {th }}$ and $f(R)$ but extremely sensitive to the radial dependence of $T_{\text {cld. }}$ A positive observational check on the above results may be made by using CFR92. The number density of a CFR cloudlet near the central object is approximately $n_{\mathrm{th}}=10^{16-18} \mathrm{~cm}^{-3}$ for an absorption column of $N_{\mathbf{H}} \leq 10^{21} \mathrm{~cm}^{-2}$ for most Seyfert 1 nuclei, while the density of the broad-line region clouds at $\sim 10^{4} R_{g}$ is $n_{\mathrm{th}} \sim 10^{10} \mathrm{~cm}^{-3}$ (e.g., Ferland \& Rees 1988). Then
we find that $n_{\mathrm{th}} \propto R^{-2}-R^{-2.66}$.

Using the $R$ dependence of the physical variables as shown above, reflection is found to be most effective at intermediate radii. At a representative radius of $R=30 R$, the solutions of equations (2), (3), (4), and (7) give $T_{\text {ctd }} \simeq 3 \times 10^{5} \mathrm{~K}$ and $n_{\mathrm{tb}} \simeq$

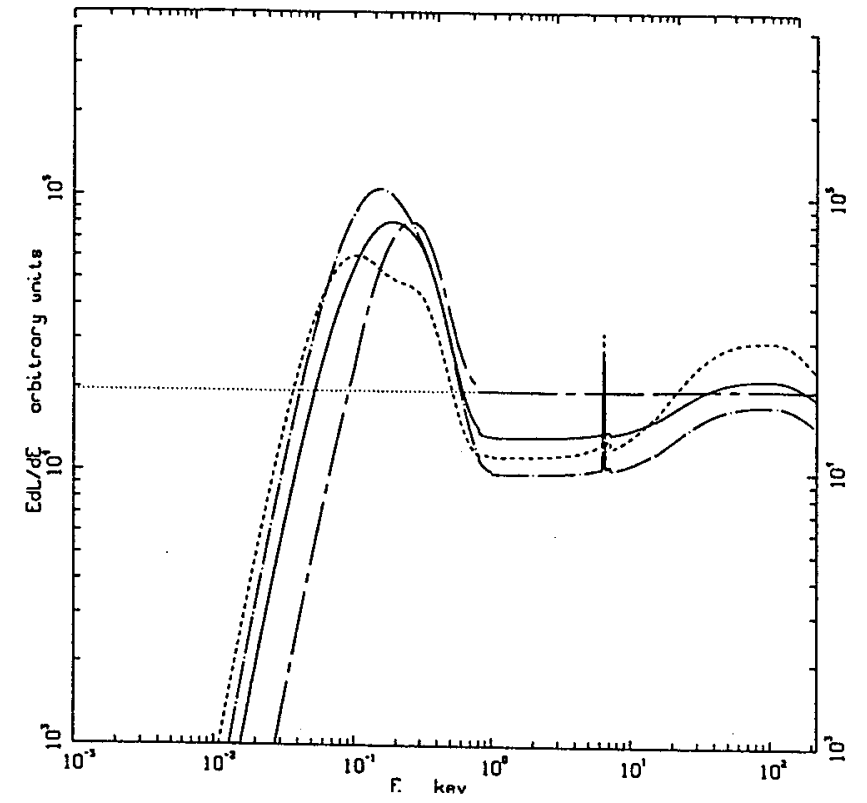

Fig. 1.-The spectra of radiation coming out of a spherical distribution of cool clouds are shown with the solid and dot-dashed curves, for the covering factor $f=0.5$ and 0.9 , respectively. The dotted curve shows the primary synchro-Compton spectrum. The chain-dashed curve is the spectrum from the innermost region of the central engine where very small CFR cloudlets are present (see the text). The dashed curve shows the spectrum coming out of a slab-corona system. The average temperature of the cool component is set at
$T_{\text {cld }}=3 \times 10^{5} \mathrm{~K}$.

$10^{16} \mathrm{~cm}^{-3}$. As our free parameters the values $L \sim 10^{43}$ ergs $\mathrm{s}^{-1}$ and $M \sim 10^{7} M_{\odot}$ are adopted, which are representative of typical Seyfert nuclei such as NGC 4051 with $L_{x}(2-10$ $\mathrm{keV})=10^{41.5}$ ergs s$^{-1}$ (e.g., CFR92; Ferland \& Rees 1988). Furthermore, with $f \sim 0.9$ the additional equation $(5)$ gives $l_{\text {cld }}=10^{12} \mathrm{~cm}$ and $n_{\text {cld }}=10^{-39} \mathrm{~cm}^{-3}$. As $v_{\text {cld }}$ the Keplerian
velocity is adopted.

As a starting point in our calculations of the outcoming spectra we assume that the average cloud temperature $T_{\text {cld }}=$ $3 \times 10^{5} \mathrm{~K}$, the average number density of the matter in the cloud $n_{\mathrm{th}}=10^{16} \mathrm{~cm}^{-3}$, and the photon index for the synchroCompton power-law spectrum $\Gamma=2$. These values are chosen to be consistent with typical Seyfert parameters obtained from observation, such as $L \sim 10^{43}$ ergs s$^{-1}$ and $M \sim 10^{7} M_{\odot}$. The lower and upper limits to the radial distance, $R_{\min }$ and $R_{\max }$, are set to $10 R_{g}$ and $1000 R_{g}$, respectively. These boundaries are determined by the distances where the $\mathrm{X}$-ray reflection ceases to be effective (ST92). The results are summarized in Figure 1. The dotted curve shows the primary synchro-Compton powerlaw spectrum. This spectrum is modified by the absorption of the IR-optical portion by the extremely small CFR cloudlets with temperature $=10^{6} \mathrm{~K}$. The modified spectrum is shown by the chain-dashed curve. Note that the absorbed IR-optical radiation is reprocessed and reappears as a hot thermal UV bump. This modified primary spectrum is used as the input to be reflected and reprocessed by an assembly of clouds. Since the clouds are optically thick in X-rays, only the unabsorbed portion, $I_{\mathrm{in}}(0) e^{-\delta\left(R_{\max }\right)}$, of the original power-law source is observed. The spectra of the total outcoming radiation obtained from equation (6d) is shown by the solid curve for the total covering factor $f \equiv f\left(R_{\max }\right)=0.5$, and by the chain-dotted curve for $f=0.9$. For comparison the dashed curve shows the 


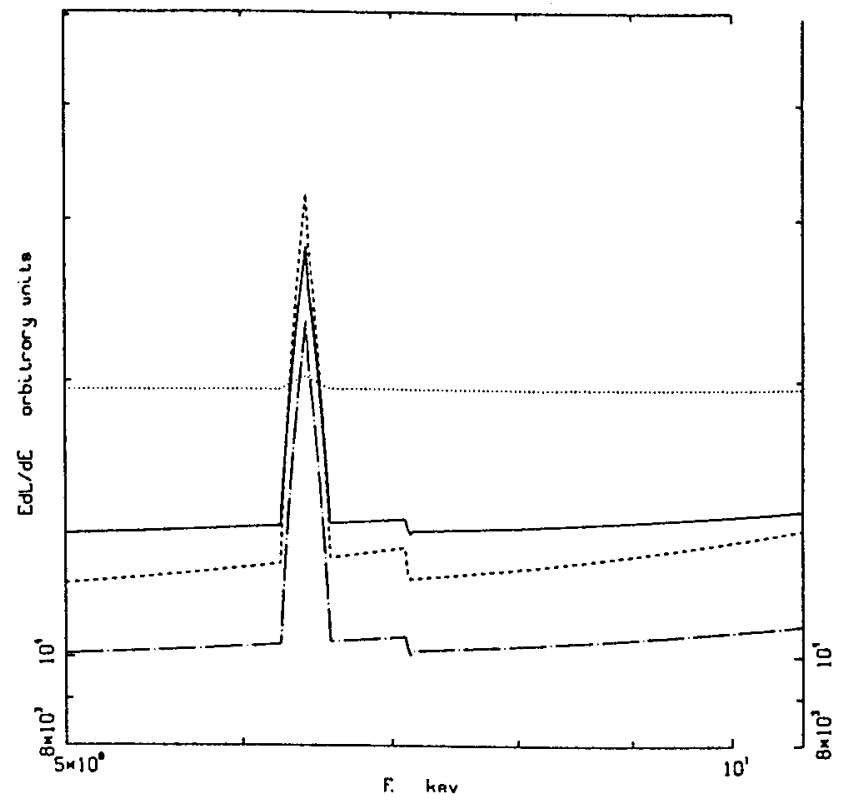

Fig. 2-- Here the $5-10 \mathrm{keV}$ section of the spectrum in Fig. 1 is enlarged. The upper dotted curve is now for $f=0.01$. Otherwise the notation is the same as in Fig. 1.

spectrum of radiation reflected from a single cold slab, using the same input spectrum (LW88). Figure 1 is enlarged in Figure 2 to show the details of the 5-10 keV X-ray region. The upper dotted curve is now the spectrum for $f=0.01$. Otherwise the notation is the same as in Figure 1. The photoelectric effect is represented by the $\mathrm{Fe} 6.4 \mathrm{keV}$ emission line and $7.1 \mathrm{keV}$ edge, for demonstrative purposes.

In Figure 1 the broadening of the UV bump extending from the UV to the soft X-ray excess region, as well as the effect of free-free absorption and Compton recoil, are clearly seen. In Figure 2 the "flattening" of the power-law spectrum from $\Gamma=2$ to $\Gamma=1.7 \pm{ }_{0.7}^{0.4}$ in the X-ray band (e.g., Zdziarski et al. 1991 ) is evident. Also noted is the increase in the equivalent width with increasing $f$. Note that the equivalent width for the cloud model with $f \sim 0.7$ is similar to that of the slab case with $f \sim 0.5$, while the Fe edge is much smaller for the cloud model. Even without the washing-out effect of the Doppler shifts, etc., the edge is not noticeable as $f$ is decreased below $\sim 0.5$.

Previous authors have found good agreement with the X-ray spectral data of some AGNs when the reflection from a single slab is adopted (e.g., LW88; George \& Fabian 1991, and references therein). Qualitatively, our model also gives similar $\mathrm{X}$-ray spectra when $0.1<f<1$. However, our cloud model is more flexible than the disk model because it generally covers a wider range of the covering factor. For instance, for the standard disk model $f$ is fixed at 0.5 . Quantitatively, with the same value of $f$ the major spectral effects of the cloud configuration, as compared with the slab geometry, are a significant increase of the UV bump and a significant decrease of the reflection features, such as the Fe line-edge system and the hard X-ray bump. The latter effect is caused by the obscuration of the reflected component by the clouds between the reflecting cloud and the observer.

Most of the covered portion of the input spectrum is absorbed by the clouds and reradiated as thermal radiation producing a large UV bump. Therefore, we calculated the total integrated bolometric luminosity for the most extensively studied rapidly variable Seyferts, NGC 4051 and NGC 6814, in order to check whether it would exceed the Eddington luminosity. It is found to be less than or roughly equal to the Eddington limit. In this calculation the X-ray portion of the spectrum was set equal to the observed X-ray intensity of these objects. Also assumed are a large $f$, e.g., $\sim 0.9$, and the black hole mass of $10^{6}-10^{7} M_{\odot}$.

\section{DISCUSSION AND CONCLUDING REMARKS}

Let us compare the variability behavior of the cloud model and the thin disk models. Intensity variations of the broad thermal UV bump produced by the clouds are temperatureand frequency-dependent. For the cloud model a "turnoff" of the central X-ray emitting source will result in cooling of the clouds. The time scale is derived from $d E / d t=-4 \pi l_{\mathrm{cld}}^{2} \sigma_{\mathrm{B}} T_{\text {cld }}^{4}$, where $E$ is the total energy of one cloud and $T_{\text {cld }}(E)=$ $3 E /\left(4 \pi l_{\mathrm{cld}}^{3} n_{\mathrm{th}} k_{\mathrm{B}}\right)$ is found from the ideal gas law. Solving this equation we get

$$
t_{c}>\frac{3 \times 10^{16}}{T_{\text {cld }}^{3}} \mathrm{~s},
$$

for Seyfert nuclei, e.g., NGC 4051 (ST92). For instance, for very soft X-rays at $T_{\mathrm{cld}}=10^{6} \mathrm{~K} t_{c} \sim 0.03 \mathrm{~s}$, while it is nearly a year for the UV radiation at $T_{\text {cld }}=1000 \mathrm{~K}$. Therefore, with our model, while the high-energy end of the bump should vary almost simultaneously with the harder X-rays, similar rapid variations are not expected at the low-energy end of the bump lying in the UV band. We carefully examined the spectral behavior of the NGC 4051 data near the $B$ band (Done et al. 1990) which shows no rapid variability during the X-ray flares, and find that the low-energy end of the UV bump already appears there. Therefore the lack of rapid variability at this wavelength is consistent with our cloud model. In our model the power-law portion of the IR-optical radiation is due to different mechanisms (Tsuruta 1990), and hence no correlation is predicted between the IR-optical-UV bands and the X-ray band. On the other hand, definite correlated variations are predicted among the IR-optical-UV and X-ray bands when a variety of disk models are adopted; for instance, the standard thin-disk model (Shakura \& Sunyaev 1977) and a disk with a hot spot (Abramowicz 1990). Simultaneous X-ray continuum and Fe line variations (within $\sim 300 \mathrm{~s}$ ) observed in NGC 4051 (Kunieda et al. 1990) are consistent with our cloud model if the line is produced at $\leq 30 R_{\theta}$, because then, with $M \sim 10^{6} M_{\odot}$ predicted for NGC 6814, the delay in the line variation should be $\leq 300$ s.

The cloud model might also be applied to galactic X-ray sources which are black hole candidates, because the underlying physical mechanisms governing these objects are considered to be similar. In the case of the galactic black hole candidate Cygnus $\mathrm{X}-1$, our cloud model agrees better with observations than disk models, because the weaker and symmetrical Fe line feature predicted from our models with $f<0.2$ is consistent with the observed data of this source, while the slab reflection models predict this feature to be stronger than and different in shape from the observed Fe line (Done et al. 1991).

Another means to distinguish between the disk and cloud models comes from the observation of polarization. In thin disks scattering atmospheres should induce a high degree of polarization (Netzer 1990, and references therein). On the other hand, our cloud model predicts very low polarization, because 


\section{SIVRON \& TSURUTA}

for the size of clouds of our kind the reflection directionality in equation (6a) results in weak polarization (ST92). Observations favor weak polarization (Netzer 1990).

No sharp Lyman edges (as predicted by many disk models) are expected to be observable with the cloud model, because the strong feature emitted by the clouds is broadened and velocities. This is the Doppler shifts with a variety of cloud Kinney, \& Ford 1989 and In reality the

sotropically, forming, most likely distributed unidue to the expected e.g., a quasi-spherical flow or a torus, Tsuruta 1990). These presence of angular momentum (e.g., investigation (ST92) but the rical effects are currently under region as spherical , but the results from treating the cloud region, except, posible still be valid in the interior of this because of the boundaries. Also, clouds is most likely na magnetic effects the shape of the or filamental. Wely nonspherical, e.g., melon-seed or sheetlike, still qualitatively adequate, because the random orientation of
and the clouds will average out most geometrical effects other than the ones considered in this paper. The possible dependence of The inclusion of the 0 of the clouds will be discussed in ST92 in an excess in soft $X$-rays when $n \sim 10^{15}-18$ edges will result $n_{\mathrm{th}} \sim 10^{15-18} \mathrm{~cm}^{-3}$, the value will result in spectral beaming, general relati These improvements

In conclusion, the existing ouded in our subsequent work. appears to favor the cloud observational evidence already models. Further careful the disk should be invaluable to theoretical and observational work

We thank M. J. Rees, K. Leighly, C. Done, T. Tanaka, J. McLaughlin, and N. Sivron for helpful discussions. We are especially greateful to D. Caditz for the careful scrutiny of the manuscript, and to the anonymous referee for the constructive comments. This work was supported partially by the NASA grant NAGW-2208 and NSF grant RII-8921978.

Abramowicz, M. A. 1990, in Structure and ERERENCES

Disks, ed. C. Bertout, $S$ Collin 299

Lightman, A. P., \& White, T. R. 1988, ApJ, 335, 738 (LW88)

Antonucci, R. R. J., Kinney, A. L., \& Ford, H. C. 1989, ApJ, 342, 64

Nandra, K., Pounds, K. A., Stewart, G. C., George, I. M., Hayashida, K. Netzer, H. 1990, in Structure 1991, MNRAS, 248, 760

C. Bertout, S. Collin, \& J.-P I Emission Properties of Accretion Disks, ed

Done, C., Ward, M. J., Fabian, A. C. 1992, MNRAS, 255, 419 (CFR92)

Smith, M. G., \& Wamsteker, W. 1990 Kunieda, H., Tsuruta, S., Lawrence, A., Done, C., Mulchaey, J. S., Mushotzky, MNRAS, 243, 713

Fabian, A. C., Blandford, R. D., Guilbert, \& Arnaud, K. A. 1991, preprint 1986, MNRAS, 221, 931

Ferland, G. J., \& Rees, M. J. 1988, ApJ, 332, 141

George, I. M., \& Fabian, A. C. 1991, MNRAS, 249, 352

Kunieda, H., Turner, M. J. 1988, MNRAS, 233, 475 (GR88)

Tsusaka, Y. 1990, Nature, 345,786 ., Koyama, K., Mushotzky, R. F., \&

Rees, M. J. 1987, MNRAS, 228, 47P (Paris: Editions Frontières), 177

Shakura, S. I., \& Sunyaev, R A, 47P

Sivron, R \& Tsuruta S R. A. 1977, A\&A, 24, 337

Tsuruta, S. 1990, in Structure in preparation (ST92)

C. Bertout, S. Collin, \& J.-P and Emission Properties of Accretion Disks, ed . 1992, in The Frontiers of (Paris: Editions Frontières), 289

Y. Tanaka (Tokyo: Univ. Acad of X-Ray Astronomy, ed. K. Koyama \& Zdziarski, A A Ghisellini, Acad. Press, Inc.), 589

Done, C. 1990, ApJ, 363, L1 